\title{
High-Speed Atomic Force Microscopy and Nanomechanical Mapping as Tools for Studying Dynamic Membrane Remodeling Processes
}

\author{
Lorena Redondo-Morata
}

Univ. Lille, CNRS, Inserm, CHU Lille, Institut Pasteur de Lille, U1019 - UMR 9017 - CIIL - Center for Infection and Immunity of Lille, F-59000 Lille, France.

Atomic force microscopy (AFM) has recently undergone significant technological improvements that enable recording of surface topography in the sub-second timescales. This development, termed HighSpeed AFM (HS-AFM) is particularly of interest for the study of the dynamics of biomolecules. It was used with great success to study the structural dynamics of molecular motors such as myosin V [1] and the characterization of membrane protein dynamics in a lipid bilayer [2]. Most recently, we have succeeded to visualize the molecular mechanism of Snf7 assembly formation [3]. Snf7 is the major polymerization component of the Endosomal Sorting Complex Required for Transport-III (ESCRT-III). We observed the formation of spiraling filaments on negatively charged membranes and estimated that these filaments can store sufficient elastic energy to drive membrane deformation. In our most recent work, we studied the molecular role of $\mathrm{Vps} 4$, an ATPase that it is known to drive the disassembly of persisting filaments of ESCRT-III [4]. We observed that in the presence of Vps4, ESCRT-III polymers disassemble partially, remaining the most-inner part of the ring-like structure refractory to the action of the enzyme. Surprisingly, in the presence of a soluble Snf7 pool, ESCRT-III assemblies shrink under the action of Vps4, liberating free space in the membrane were new ESCRT-III assemblies are growing simultaneously (Figure 1). This results in a high exchange and lateral mobility of ESCRT-III assemblies on membranes. Dynamic exchange provides an explanation for how ESCRT-III filaments gradually adapt their shape during membrane constriction, which has broad implications in diverse cellular processes, differing in size, shape, and duration - such as plasma membrane repair, cytokinesis, or viral budding.

Using similar methodology, we are also interested in the dynamic nanomechanical changes of the lipid membrane due to lipid-lipid interactions. In lipid membranes, the ultimate lipid phase coexistence to be fully understood are transient nanodomains, often (confusedly) referred to as lipid rafts [5]. Based on the current knowledge, microdomains in equilibrium are no longer considered suitable models for the biological structure that rafts represent. Multiscale spatiotemporal measurements of the membrane mechanical properties can help to experimentally address different scenarios where membrane micro- and nano-domain formation finds theoretical support. Atomic Force Microscopy (AFM)-based Force Spectroscopy can resolve coexistence of domains at concentrations where height differences at domain boundaries are not detectable [6]. Non-equilibrium fluctuations by tuning membrane (local and global) environment actively controlling curvature, leaflet asymmetry, solid support topography and addition of external components would help complete the manifold where domain formation occurs. Here, we will focus on the in situ conversion of sphingomyelin to ceramide. Ceramide is produced in cells from sphingomyelin by means of the enzymatic activity of endogenous sphingomyelinase. The presence of ceramide has a high impact in the physical chemical properties of the membrane, inducing changes in the curvature, phase, segregation, and order. The local production of ceramide induces local mechanical changes depending on the chain length and degree of unsaturation of the original sphingomyelin (Figure 2). AFM-based Force Spectroscopy is an ideal technique to investigate the mechanical properties of lipid bilayers at the nanoscale, their elastic constants [7] but also their plastic deformation and rupture [6]. However, the viscoelastic parameters of lipid membranes have been less explored by these means. In this 
work, we systematically studied the enzymatic conversion of sphingomyelin-containing supported lipid bilayers to ceramide by adding sphingomyelinase in situ. The different ceramide localization in the membrane and mechanical properties is relevant in several biological contexts as apoptosis or viral infection. Finally, we will discuss possible approaches to evaluate the dynamic changes in viscoelasticity of lipid membranes [8], i.e., using fast mapping with bimodal AFM [9].

[1] N Kodera et al, Nature 468 (2010), p. 72.

[2] I Casuso et al, Nature Nanotechnology 7 (2012), p. 525.

[3] N Chiaruttini et al, Cell 163 (2015), p. 866.

[4] B Mierzwa et al, Nature Cell Biology 19 (2017), p.787.

[5] FM Goñi, Chemistry and Physics of Lipids 218 (2019), p. 34.

[6] L Redondo-Morata et al, Langmuir 28 (2012), p. 12851.

[7] L Redondo-Morata et al, Biophysical Journal 111 (2016), p. 363.

[8] Z Al-Rekabi, S Contera, Proceedings of the Natural Academy of Sciences 115 (2018), p. 2658.

[9] V Gisbert et al, ACS Nano 15 (2021), p. 1850.

[10] The author acknowledges funding from the Agence National de la Recherche (ANR), as part of the 'Investments d'Avenir' Programme (I-SITE ULNE / ANR-16-IDEX-0004 ULNE).
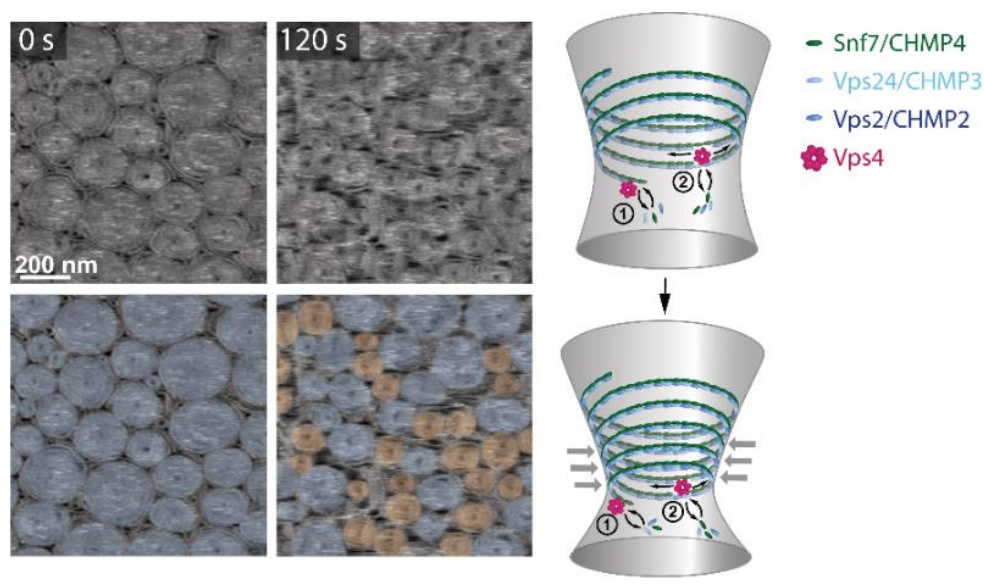

Figure 1. HS-AFM imaging of ESCRT-III polymers on supported lipid membranes, followed by the addition of Vps4 and ATP. The overlays highlight pre-formed spirals (blue) or newly formed spirals (orange). Our model suggests that Vps4 induces dynamic reorganization of ESCRT-III assemblies.

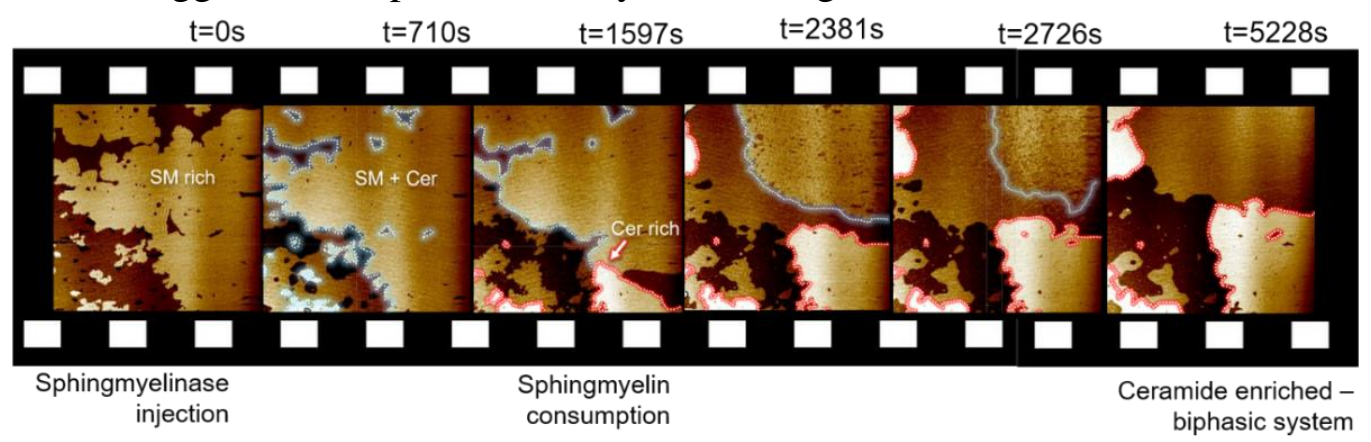

Figure 2. Fast nanomechanical mapping of a biphasic lipid membrane. After injection of sphingomyelinase to the solution, sphingomyelin-enriched domains evolve to ceramide-enriched domains, which impacts the lipid order, domain distribution and local mechanics. 